\title{
Ciclo de Mejora en un aula de Asesoramiento Curricular y Competencias Básicas en Lengua, Literatura y Matemáticas
}

\section{Improvement Cycles in Classroom of Curricular Advice and Basic Competences in Language, Literature and Mathematics}

ANA ESCUDERO-DOMÍNGUEZ

ORCID: https://orcid.org/0000-0002-1620-3852

Universidad de Sevilla

Departamento de Didáctica de la Matemática Aescudero1@us.es

Fecha de recepción: 06-11-2019

Fecha de aceptación: 17-11-2019

DOI: http://dx.doi.org/10.12795/9788447221912.084

Pp.: 1907-1928 


\section{Resumen:}

Se presenta un ciclo de mejora en un aula de 40 del Grado de Pedagogía, concretamente en la asignatura de Asesoramiento Curricular y Competencias Básicas en Lengua, Literatura y Matemáticas. Nosotros nos centraremos solamente en la parte matemática. El objetivo de este trabajo es la introducción de un cambio metodológico en la enseñanza de la asignatura, planteando la posibilidad de que los alumnos aprendan parte de los contenidos a partir de la resolución de diversos casos con ejemplos del mundo real. Finalmente, la evaluación del nivel de aprendizaje de los estudiantes se refleja mediante el uso de escaleras de aprendizaje. Los resultados muestran una tendencia positiva, tanto en las valoraciones del alumnado como las del profesorado. Por otro lado, las dificultades halladas y el análisis de la intervención sirven como base para futuras propuestas de mejora en el diseño.

Palabras clave: Asesoramiento Curricular y Competencias Básicas en Lengua, Literatura y Matemáticas, Grado en Pedagogía, Docencia universitaria, Experimentación docente universitaria

\section{Abstract:}

A cycle of improvement is presented in a classroom of 40 of the Degree of Pedagogy, specifically in the subject of Curricular Advice and Basic Competences in Language, Literature and Mathematics. We will focus only on the mathematical part of the subject. The objective of this work is the introduction of a methodological change in the teaching of the subject, raising the possibility that students learn part of the contents of the subject from the resolution of various cases with real-world examples. Finally, the evaluation of the students' level of learning is reflected through the use of learning ladders. The results show a positive trend, both in the assessments of students and teachers. On the other hand, the difficulties encountered and the analysis of the intervention serve as the basis for future proposals for improvement in the subject.

Key words: Curriculum Counseling and Basic Competences in Language, Literature and Mathematics, Degree in Pedagogy, University Teaching, University Teaching Experimentation 


\section{Contexto de la asignatura}

La Asignatura de Asesoramiento Curricular y Competencias Básicas en Lengua, Literatura y Matemáticas es una asignatura optativa del último curso del Grado de Pedagogía, que comparten el departamento de Didáctica de las Matemáticas y el departamento de Didáctica de la Lengua y la Literatura y Filologías Integradas. En esta los alumnos se enfrentan por primera vez en el Grado a algunas nociones matemáticas. El enfoque de esta asignatura es la profesionalización del pedagogo. Sabemos que el perfil del pedagogo no está definido, pero con la aparición de los departamentos de orientación surge un perfil profesional con puesto de trabajo claro tanto en enseñanza reglada como no reglada. El número total de alumnos matriculados en la misma es de 56 personas, de los cuales acudían a clase una media de 40. Las condiciones de espacio eran adecuadas, ya que se trataba de una clase amplia, donde las mesas no estaban ancladas al suelo, lo que facilitaba el trabajo en grupo.

Es importante destacar que, aunque es la única asignatura que les va a ofrecer alguna pincelada matemática en todo el grado, los estudiantes no están, por lo general, motivados. El primer día en una presentación no oficial de estos, les pregunté qué porqué habían elegido realizar esta asignatura y la mayoría me respondió que la habían elegido por cuestión de horario, no por interés. Además, muchos de ellos expresaban una actitud negativa hacia las matemáticas. Dentro de nuestra parte de la asignatura vamos a intentar abarcar las emociones y creencias acerca de las matemáticas.

\section{Diseño del CIMA}

Este año cambio de titulación y asignatura para llevar a cabo el ciclo de mejora en el aula (en adelante, CIMA). Este curso tomaré toda la parte de matemáticas de la 
asignatura Asesoramiento curricular y competencias básicas en lengua, literatura y matemáticas, una asignatura optativa de 4o curso del grado de Pedagogía, que abarca las 7 primeras semanas del primer cuatrimestre.

La asignatura tiene establecido horario de clases teóricas y clases prácticas, sin embargo, nosotros no vamos a hacer distinción entre ellas.

Esta parte de la asignatura está organizada en 3 bloques de contenidos, uno centrado en el curriculum, otro en aprendizaje y otro en enseñanza, es decir, teniendo en cuenta el triángulo contenido matemático, alumno y profesor. Con esta organización intentamos dar respuesta a 3 grandes interrogantes: ¿Qué enseñar?, ¿Cómo enseñar? y ¿Para qué enseñar?

Tratando los 3 bloques anteriormente mencionados vamos a trabajarlos a través de distintos talleres. Tenemos una media de 2 semanas para trabajar cada bloque de contenidos. En estos se lanzarán una serie de preguntas que intentaremos ir respondiendo entre todos, ya que mi intervención pretende llevar a cabo un modelo didáctico de aprendizaje significativo, por reestructuración. Vamos a evitar la exposición de lecciones magistrales (Bain, 2004), proponiendo que sean los estudiantes los que construyan el conocimiento, cediéndoles la responsabilidad (Carrillo, Climent, Gorgorió, Prat y Rojas, 2008). Para ello nos basamos en el capítulo 6 del libro de Finkel "Dar clase con la boca cerrada" (Finkel, 2008) en el que se describe una experiencia en la que un profesor va guiando a los alumnos a través de preguntas encadenadas para resolver un determinado problema. Estas preguntas ayudan a los estudiantes a organizar y facilitar sus razonamientos pero sin llegar a proporcionarles la respuesta directa.

Para poner en práctica el "dar clase con la boca cerrada" diseñamos unos casos (con ejemplos del mundo 
real) donde poner en práctica la teoría que se pretende enseñar. Los estudiantes trabajarán sobre estas cuestiones en pequeño grupo, poniéndose en situación (pues los protagonistas de los casos son recién titulados en Pedagogía, por lo que pueden sentirse identificados) y tienen una serie de problemas que deben solventar. Para ello, las preguntas les servirán de guía para que los alumnos piensen por sí mismos y encuentren la solución a estas dificultades o entiendan por qué se producen. A la finalización-resolución de cada caso se pondrán en común con el resto de grupos y con el docente, por lo que se recibirá un feedback.

Para la definición del modelo metodológico usado en este ciclo de mejora, retomo algunas de las conclusiones aportadas en ciclos de mejora realizados anteriormente, siendo el modelo usado en mis clases el siguiente:

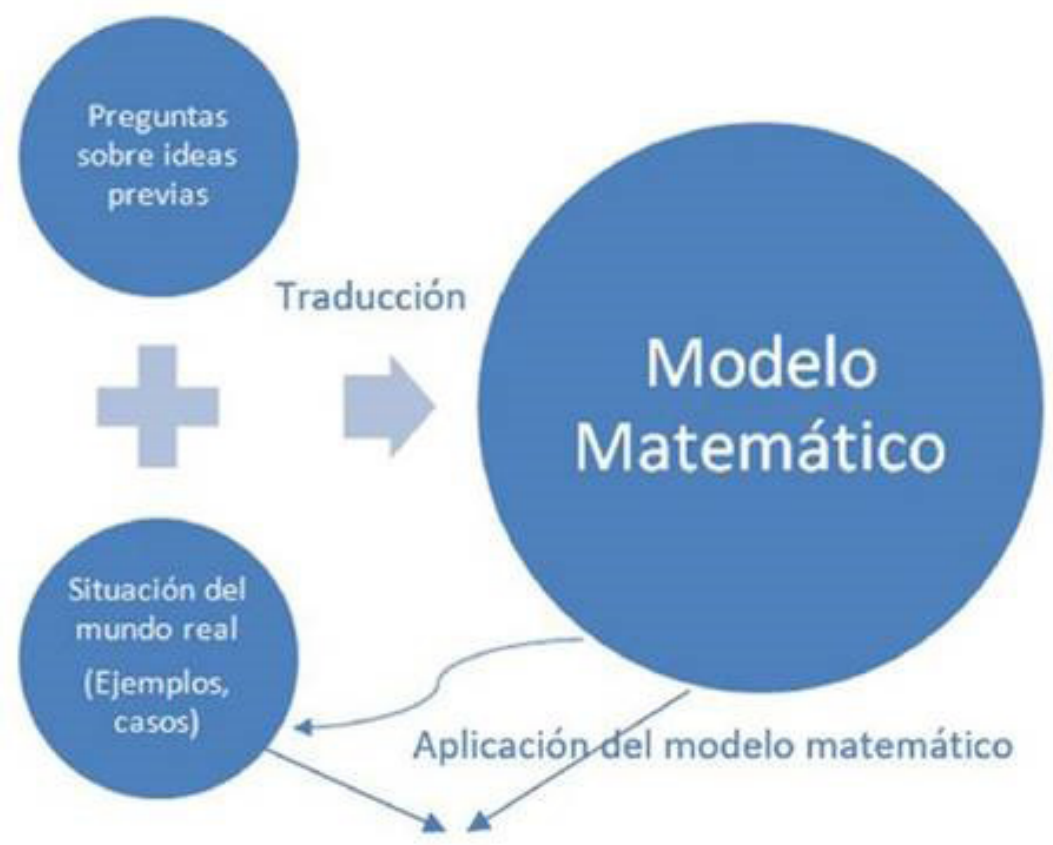

Aprendizaje final

Figura 1. Modelo metodológico llevado a cabo

Jornadas de Formación e Innovación Docente del Profesorado I № 2 (2019) Esta obra se distribuye con la licencia Creative Commons Reconocimiento-NoComercial-SinObraDerivada 
Por lo que la forma de trabajo que se propone es la siguiente:

Se comienza el bloque realizando preguntas para conocer de donde parten los estudiantes, obteniendo así las ideas previas de estos alumnos. A continuación, se les van planteando distintas preguntas encadenadas que estructuran el contenido a tratar. Esto lo realizarán en pequeño grupo, de unos 4-5 estudiantes. Se les pide que vayan buscando respuesta a las distintas preguntas que se les plantean, ya sea con material audiovisual o buscando otros materiales como lecturas, libros de textos y/o transcripciones de un caso real. A partir de lo anterior pretendemos producir una traducción a lo abstracto, es decir, a lo que sería el modelo matemático (teórico). Para ello se ayudarán de nuevo de los ejemplos tratados anteriormente como aplicación del modelo matemático, a partir de las lecturas recomendadas. Finalmente, se pondrán en común mediante una exposición corta. También deberán colgar su trabajo en la plataforma, de forma que todos los alumnos tengan acceso al material elaborado. Este último proceso nos conduce al aprendizaje final, es decir, pretendemos hacer partir al alumnado de situaciones reales, para que creen un modelo de ese mundo real y luego que sean capaces de extrapolarlo a un modelo matemático, generando unas conclusiones al respecto, apoyadas en el modelo matemático existente. Esta metodología favorecerá en el alumno la capacidad para aprender por sí mismo.

\section{Mapa de contenidos}

Los contenidos que voy a abordar en este CIMA pretenden abarcar tres pilares (conocimiento matemático, aprendizaje y enseñanza). Años anteriores me habría obsesionado con dar bastantes apartados dentro de cada bloque de contenidos, pero he aprendido que no se puede dar todo. Además, en la actualidad existe un acceso rápido y fácil a la información, por lo que en lo que tenemos que 
esforzarnos es en reflexionar sobre cuáles son las preguntas o contenidos organizadores que dan sentido y ayudan a explicar los distintos aspectos de la temática de estudio (Escudero-Domínguez, 2017).

Como de costumbre en el departamento solemos dividir cada bloque de contenidos con tres grandes preguntas. Esta asignatura la hemos dividido en tres bloques de contenidos, uno dedicado a cada una de las partes del triángulo didáctico.

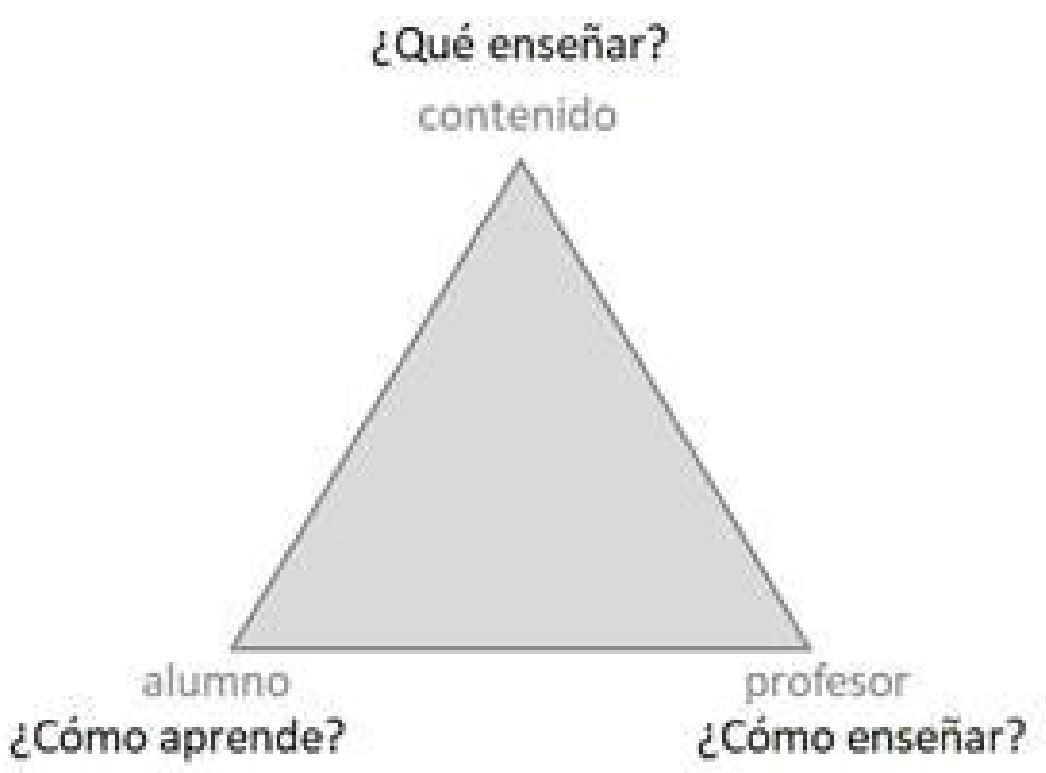

Figura 2. Triángulo didáctico

Comenzamos con el bloque sobre contenido, que abarcará desde el 24 de septiembre al 8 de octubre, en el que se abarcará, entre otras, las preguntas ¿Qué enseñar? ¿Por qué se debe enseñar matemáticas? Además de esto sabemos que con el curriculum se debe dar respuesta a los fines, por lo que lanzaremos esta otra pregunta: ¿se pueden apreciar diferentes fines en educación matemática? Para terminar, lanzamos esta última pregunta: ¿pueden existir distintas formas de considerar el curriculum matemático? 
El siguiente módulo, que comenzaremos el 9 octubre, trabaja al aprendizaje y, por tanto, como principal pregunta ¿Cómo aprende el alumno? En este bloque partimos de que no todas las situaciones matemáticas son análogas. Por tanto, existen distintos errores y estos se pueden clasificar. Lo importante es saber que la interpretación y análisis de los errores cometidos en la enseñanza-aprendizaje de las matemáticas puede enriquecerse con el apoyo de algunas teorías de la psicología educativa, ya que se ha centrado el interés al tratar de explicar la forma en que se produce el aprendizaje que ha ido variando, pasando de planteamientos centrados en lo que se aprende a aquellos centrados en cómo se aprende.

Por último, vamos a trabajar el módulo de la enseñanza, que abarcará desde el 23 octubre hasta el 6 de noviembre, queriendo dar respuesta a la pregunta ¿Cómo enseñar?. En este bloque nos vamos a centrar en los organizadores curriculares. Esto lo vamos a llevar a cabo a través de la pregunta: ¿Es posible encontrar otros elementos distintos de los contenidos que expresen un conocimiento útil y objetivo para la elaboración de unidades didácticas?, encadenando otras cuestiones que les hagan cuestionarse algunos aspectos a los estudiantes, a la vez de orientarlos hacia la respuesta.

A continuación, se muestra, en primer lugar, el esquema general usado para la asignatura, y en segundo lugar, el mapa de contenidos con las preguntas estructurantes perteneciente al módulo 1:

Jornadas de Formación e Innovación Docente del Profesorado | № 2 (2019) Esta obra se distribuye con la licencia Creative Commons Reconocimiento-NoComercial-SinObraDerivada 4.0 Internacional (CC BY-NC-ND 4.0.) 


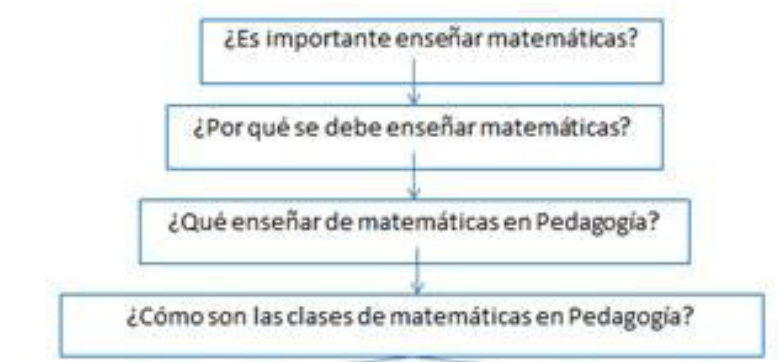

Memoristicas
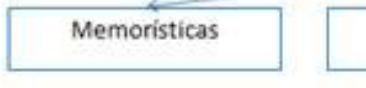

¿Hay otra forma de enseñar matemáticas a pedagogos?

A través de ejemplos del mundo real

Figura 3. Mapa general de la asignatura ((basado en mapa general de contenidos de Escudero_Domínguez, 2018, p. 221))

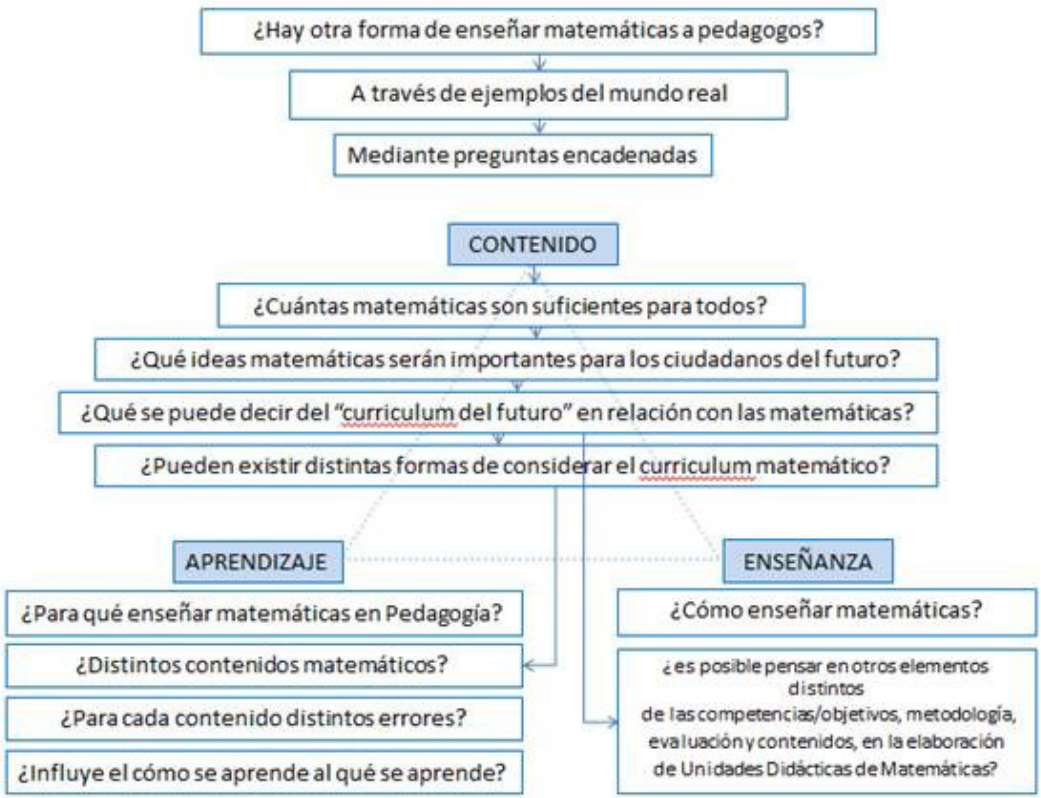

Figura 4. Mapa de contenidos y preguntas estructurantes del módulo 1

\section{Diseño de casos que estructuran los distintos contenidos a enseñar}

Tras la elaboración del mapa de contenidos con las ideas-preguntas estructurantes de cada bloque, pasamos

Jornadas de Formación e Innovación Docente del Profesorado | № 2 (2019) Esta obra se distribuye con la licencia Creative Commons Reconocimiento-NoComercial-SinObraDerivada Internacional (CC BY-NC-ND 4.0.) 
a lo que es el diseño de cada caso, que irá encadenando las distintas preguntas con el objetivo de facilitar sus razonamientos. Tratamos de no dar la respuesta sino de que ellos por si mismos sean capaces de llegar al conocimiento, mediante la ayuda de estas preguntas estructurantes (Finkel, 2008). A continuación, por cuestión de formato, solo voy a mostrar el caso 1 planteado para el módulo 1 de contenidos. El módulo 2 (aprendizaje) y el módulo 3(enseñanza) seguirían el mismo esquema.

Los casos contienen una situación real de una o varias personas que acaban de terminar el Grado de Pedagogía y recientemente se han incorporado al mercado laboral. En estos, según el bloque de contenidos que se quiera abarcar, se plantean algunos problemas que invitan a los alumnos a trabajar sobre ellos en pequeño grupo, reflexionando en un primer momento desde sus ideas previas, lo que sería como el cuestionario inicial. Después de ello, se les facilita una serie de materiales, ya sean audiovisuales, lecturas de investigación o libros de texto, a partir de los cuales y, guiados a través de preguntas encadenadas, se pueda lograr acercar a los alumnos a la búsqueda de la respuesta de las distintas cuestiones que se plantean (cuestionario final). Los estudiantes harán entrega al final de cada módulo de un informe por grupo de trabajo, donde vendrán recogidas las respuestas iniciales (ideas previas) y las respuestas finales. Estas actividades serán analizadas y utilizadas para la evaluación, pues ahí se observa la evolución de las ideas de los estudiantes con respecto a los contenidos que se pretenden enseñar. Al inicio de cada caso se recogerá información sobre el punto de partida en el que se encuentra el alumnado, siendo de esperar que, tras el ciclo de mejora, el conocimiento del alumnado haya mejorado. 


\section{Cuestionario Caso 1_Módulo 1}

Juan y Ana acaban de terminar Pedagogía. Han sido compañeros de carrera y, a lo largo de ella, en muchas ocasiones han formado parte de un mismo grupo de trabajo, por lo que están acostumbrados a compartir tareas y preocupaciones.

Ana ha encontrado su primera salida laboral haciendo una sustitución como orientadora en un centro escolar de Secundaria. Transcurridas dos semanas, se reúnen para hablar de cómo les ha ido. Ana está desanimada. La primera semana asistió a una reunión del Consejo Escolar y salió descorazonada. Los padres de los alumnos estaban preocupados por los bajos rendimientos en Matemáticas y los resultados de informes divulgados en la prensa como el informe PISA, y los profesores de Matemáticas exponían sus argumentos. El debate era acalorado, y parecía que subyacian puntos de vista muy distintos sobre por qué y para qué se debía estudiar matemáticas, pero ella no intervino ni una sola vez. Nadie contaba con ella, y no parecía tener ningún papel en aquella reunión. Pero lo más grave era que tampoco tenía nada que decir. Ni siquiera cuando algunos basaban sus argumentos en las propias finalidades de la enseñanza de las Matemáticas.

La verdad es que su experiencia personal con esta materia no había sido buena, y guardaba un pésimo recuerdo de esta signatura de sus años escolares, pero ella no era ahora alumna, ni formaba parte del colectivo de padres, ni del de profesores de Matemáticas. ¿No había absolutamente nada, desde la perspectiva de pedagoga asignada a un Departamento de Orientación, que pudiese aportar a este debate?

Tampoco Juan estaba muy satisfecho. Él colaboraba con un equipo de orientación interdisciplinar pero no lograba que sus intervenciones de apoyo y asesoramiento fueran tenidas en cuenta por los profesores de Matemáticas. Hace dos días estuvo en una reunión con profesores de esa asignatura para hablar de planificación, en 
particular de cómo concretar en el Proyecto Curricular de sus diferentes Centros los objetivos, contenidos, metodología, etc., explicitados en los Documentos oficiales. Él quería que se tuviese en cuenta que se estaban preparando los ciudadanos del futuro, pero no conseguía argumentar claramente lo que esto implicaba. También tuvo dificultades para intervenir en la acalorada discusión que se desencadenó sobre cómo se deberían incorporar las nuevas tecnologías en las aulas de Matemáticas.

Ana y Juan han ido recogiendo en un papel algunas de las preguntas que han ido surgiendo en su conversación, y deciden buscarles respuesta. Se plantean reconstruir inmediatamente su grupo de trabajo. Es más, se comprometen a pedir ayuda a algunos profesores de la Facultad, para que les proporcionen documentos que puedan servir para comenzar a trabajar, y a buscar por ellos mismos en la Biblioteca información que les pueda ser útil. Y quedan para el viernes que viene, en el mismo sitio. Pero se les ha hecho muy tarde, y se despiden tan deprisa que dejan encima de la mesa su lista de preguntas ¿Podríamos nosotros contestarlas?

1. ¿Por qué se enseña Matemáticas?

2. ¿Se pueden apreciar diferentes fines (o finalidades) en la Educación Matemática?

3. ¿Pueden existir distintas formas de considerar el currículo matemático?

4. ¿Qué se puede decir sobre el 'curriculum del futuro' en relación con las Matemáticas?

\section{Aplicación del CIMA}

El inicio del CIMA coincide con el inicio de la asignatura y del curso. Hemos contado con una dedicación de 7 semanas. A continuación, se expone el diario confeccionado por sesiones donde se irá detallando lo tratado cada día, para facilitar la lectura y posterior evaluación del CIMA. Primera semana (24-25 septiembre): 
En la primera sesión, presentamos la asignatura y refrescamos algunos conceptos clave para, a posteriori, adentrarnos en el primer módulo. Para ello, comenzamos por una lluvia de ideas y la presentación del primer caso a trabajar, hilo conductor en las siguientes sesiones, profundizando en cada una de ellas sobre un aspecto. En esta primera sesión responden por grupo con sus ideas previas a las preguntas que en el caso se plantean (cuestionario inicial, modulo 1). Esto es entregado a la finalización de la clase. En este los alumnos quieren buscar información acerca de cada una de las preguntas, por lo que le repito en varias ocasiones que se trata de sus ideas previas al respecto, todo porque están acostumbrados a buscar la idoneidad de sus respuestas.

En la segunda sesión, comenzamos, de nuevo, leyendo el caso e intentando responder a una primera pregunta ¿Por qué se debe enseñar Matemáticas? Y tras las respuestas iniciales de los alumnos, se comienza a profundizar sobre ellas mediante algunas preguntas que intentan guiar, realizando primero una búsqueda mediante distintos recursos, para finalizar con una lectura que les lleva a construir una respuesta sólida a esta pregunta. En general, observamos cierta dificultad en la lectura y su comprensión, así como el enfrentarse a dar respuesta a la pregunta con estos recursos disponibles.

Segunda semana (1-2 octubre):

Tercera y cuarta sesión. Comenzamos cada sesión repasando brevemente lo trabajado en la anterior y, siguiendo el mismo esquema, tomando el caso y planteando dar respuestas a nuevas preguntas como: ¿Se pueden apreciar diferentes fines (o finalidades) en la Educación Matemática? mediante unas subpreguntas (preguntas que intentan desmenuzar la pregunta clave), encauzando la respuesta de los estudiantes. De estas sesiones resaltar que hay que repetir la instrucción de los pasos a seguir para la elaboración del informe, es decir, concretar cómo deben cumplimentar 
las distintas cuestiones que aparecen en el informe a partir de los distintos recursos que se trabajan. En esta semana comenzamos a exponer brevemente por grupo, de forma voluntaria, los resultados que iban obteniendo con respecto a la primera y segunda pregunta. Esto resulta interesante, pues así se abre inconscientemente un debate sobre la idoneidad de las respuestas (e.g. "pues nosotros hemos puesto esto", “y no podría ser esto también?”).

Tercera semana (8-9 octubre):

En la quinta y sexta sesión se trabajan las últimas preguntas del primer módulo: ¿Pueden existir distintas formas de considerar el currículo matemático?, ¿Qué se puede decir sobre el 'curriculum del futuro' en relación con las Matemáticas? Llevando la sesión de forma similar a las anteriores, observando, mediante las respuestas iniciales, como poder orientarlos hacia lo que sería la respuesta definitiva. En esta sesión hay varios grupos dispuestos a contarnos lo que habían contestado en sus preguntas, ya que dicen que así corregen el caso antes de entregarlo, lo que nos hace pensar en la dificultad que poseen al plasmar los resultados alcanzados.

\section{Cuarta semana (15-16 octubre):}

En la séptima sesión se repasan algunos conceptos clave de dicho módulo y se hace entrega del informe por grupos con las respuestas iniciales (con sus ideas previas) y finales (tras haber trabajado el módulo) del caso (cuestionario inicial y final caso 1), que serán las que nos ayudarán a observar la evolución de las ideas de los estudiantes con respecto a los contenidos que queríamos enseñar.

En la octava sesión comenzamos el segundo módulo que versa sobre el aprendizaje. Se trabaja de forma similar al anterior, es decir, mediante la presentación de un caso, una situación del mundo real en la que, a partir de una 
serie de preguntas, se va reflexionando y construyendo el conocimiento que queremos que adquieran al respecto. En dicha sesión los alumnos responden a las preguntas planteadas en el caso con sus ideas previas. Al igual que en el módulo anterior, se realiza en grupo, dando lugar al cuestionario inicial y se hace entrega al final de la sesión de clase. La profesora intenta mirar estos cuestionarios iniciales para el próximo día, con el objetivo de comenzar la clase a partir de preguntas orientadas al nivel de conocimientos que posee el grupo clase. Esto es complejo, pues aunque ya tiene una serie de preguntas diseñadas, hace que tenga que rehacer en poco tiempo estas preguntas y ajustarlas a sus conocimientos.

\section{Quinta semana (22-23 octubre):}

Novena sesión. En esta sesión se aborda la primera pregunta del caso 2 , en la que intentamos que profundicen acerca de las dificultades, obstáculos y errores en el aprendizaje de las matemáticas en la Educación Secundaria. De forma semejante al módulo anterior planteamos una pregunta clave que se afrontará mediante unas preguntas más concretas que los van acercando a la respuesta final. Como en clases anteriores, aunque en menor medida, nos encontramos a grupos en los que nos dicen "nosotros nos hemos leído esto... pero ¿ahora qué tenemos que hacer? Un resumen?" Lo que muestra la dificultad de estos a la hora de elaborar su respuesta. Aquí, de nuevo, comento que la planificación de este trabajo no puede consistir en repartirse la pregunta y buscar qué lectura le corresponde, sino que aquí todas las lecturas van integradas y sirven para dar respuesta a cada una de las preguntas planteadas. Sobre el final de la sesión, como en cada clase, se expone brevemente, de forma voluntaria, los resultados obtenidos. En este caso la participación es muy amplia y se propician discusiones sobre el tema tratado. Pienso que debido a que les resulta más cercano, ya 
que se trata del aprendizaje de las matemáticas (dificultades, obstáculos y errores).

Décima sesión. En esta sesión seguimos trabajando el módulo, profundizando sobre las distintas preguntas planteadas en el caso y rellenando el dossier a entregar en la próxima sesión. Se abarca aquí, además de los componentes del aprendizaje de las matemáticas, el tema de las creencias de los estudiantes, es decir, cuestiones afectivas.

Sexta semana (29-30 octubre):

En la undécima sesión terminamos el segundo módulo, mediante un repaso de algunos conceptos clave y se hace entrega del informe por grupos del caso 2 donde vienen recogidos tanto el cuestinario inicial como el final.

En la duodécima nos adentraremos ya en el último caso, para trabajar la enseñanza. Para ello hemos elegido una unidad didáctica de 10ESO sobre números, concretamente, sobre divisibilidad. Y reflexionamos sobre la siguiente cuestión: ¿Es posible pensar en otros elementos distintos de las competencias/objetivos, metodología, evaluación y contenidos, en la elaboración de Unidades Didácticas de Matemáticas? Esto lo van plasmando por escrito, en grupo, en el dossier, dejando así constancia de sus ideas previas al respecto (cuestionario inicial). Esto como en los otros dos bloques anteriores, es entregado al final de la clase. Al igual que en los anteriores, la profesora se los mira para tener un primer contacto con las ideas previas que poseen sus alumnos y así poder rehacer, reajustar las subpreguntas planteadas para resolver el caso.

Séptima semana (5-6 noviembre):

En la decimotercera sesión de clase se sigue trabajando sobre el módulo 3, profundizando sobre la pregunta planteada e intentando encauzar mediante preguntas y 
lecturas lo que son los organizadores del currículo de matemáticas. Como de costumbre, algún grupo expone los resultados obtenidos y se propicia el debate entre los distintos grupos, lo que resulta enriquecedor para todos.

En la decimocuarta sesión se entrega el informe del caso 3 (cuestionario inicial y final) y se hace una reflexión de los avances que ha tenido el grupo clase con respecto a los temas abordados, dejando la última parte de la sesión para que puedan hablar sobre qué le ha parecido la asignatura y que hagan una evaluación de mi docencia, con la idea de que me pueda ayudar para el próximo curso.

Además de recoger el diario, a modo general en este punto vamos a reflexionar sobre cómo pensamos que ha funcionado el CIMA llevado a cabo. En primer lugar, podemos decir que se ha desarrollado de forma amena y sin complejidad, dado que también se nota que son alumnos de 4으 de Grado. El plantear los distintos casos nos ha servido y nos ha sido muy útiles a la hora de abordar los contenidos de una forma distinta a la memorística y a través de clases magistrales. Aunque ha sido todo un reto el considerar qué tipo de problemas iban a ser planteados en el aula, ha merecido la pena ya que han provocado la curiosidad de éstos, poniendo sus mentes a funcionar. El plantear problemas nos ha supuesto algunos problemas a la hora de abarcar la teoría que se pretendía enseñar, además del tiempo invertido en la programación y diseño de la secuencia de preguntas estructurantes que abordar en torno al problema. En un par de ocasiones, alguna de las preguntas planteadas no ha alcanzado el objetivo planteado y tuve que buscar, reformular la pregunta para que diera pie al objetivo propuesto. En este CIMA se ha evitado la exposición de contenido por parte de la profesora, aunque los alumnos sí que han podido acudir a ésta y se ha puesto su razonamiento a disposición del alumnado. También se ha detectado cierta dificultad en la comprensión de algunas lecturas y el cómo enfrentarse a dar respuesta 
a las distintas preguntas con los recursos disponibles. He de confesar que estoy dándole vueltas a los materiales de consulta pues considero que podría ampliar o cambiar alguno de ellos.

\section{Evaluación del CIMA}

La evaluación nos permite observar la evolución del conocimiento que los estudiantes van desarrollando (Escudero-Domínguez, 2018). La evaluación de esta asignatura se realiza atendiendo a un dossier de trabajo por grupos donde los estudiantes han ido plasmando el trabajo realizado para cada caso. Este consta de una primera parte sobre ideas previas (cuestionario inicial) y una segunda con las respuestas finales a estas preguntas planteadas en dicho caso (cuestionario final). Además de esto, en la última sesión, se les pidió que redactasen, también por grupo, sus impresiones sobre la práctica, planteando las ideas que les habían resultado más interesantes, además de mostrar aquellos aspectos que cambiarían. Con esto último buscaba que hicieran una valoración que me sirviera a mí como docente.

\section{Evaluación del aprendizaje de los estudiantes. Escaleras de aprendizaje}

La evaluación del aprendizaje del alumnado durante el CIMA se va a valorar mediante la representación gráfica de las escaleras de aprendizaje (Porlán., 2017). Las escaleras se elaboraron dando respuesta a las preguntas estructurantes de cada caso antes de comenzar el ciclo de mejora y al finalizarlo. Por cuestión de tiempo y volumen de informes vamos a mostrar solo las escaleras de aprendizaje del primer módulo, pues no se ha podido completar todo el análisis. 
Las respuestas de los estudiantes se clasificaron según la adecuación a la respuesta prototipo ideal. Se establecieron distintos niveles de aprendizaje y cada respuesta se hizo corresponder con uno de ellos. Una vez elaborada la escalera, se calculó el porcentaje de grupo de alumnos que había en cada nivel. La entrega de estos cuestionarios iniciales nos permitió conocer en qué partes teníamos que insistir más, según las ideas previas de los alumnos.

En las figuras 5, 6, 7 y 8 se muestran las escaleras de aprendizaje para todas las preguntas estructurantes del módulo 1, con los patrones de respuesta que constituyen cada nivel y el porcentaje de alumnos que se sitúan en cada uno de ellos, tanto al inicio como al final del CIMA.

\begin{tabular}{|c|c|c|c|}
\hline & I: $7 \%$ & $\begin{array}{l}\text { l: } 3 \% \\
\text { F: } 90 \%\end{array}$ & $\begin{array}{l}\text { N3: Reconoce que las matemáticas sirven para desenvolverse en la } \\
\text { vida, para el estudio de otras ciencias y para preparar a ciudadanos } \\
\text { productivos. }\end{array}$ \\
\hline & F: $10 \%$ & $\begin{array}{l}\text { N2: Recor } \\
\text { sirve para }\end{array}$ & $\begin{array}{l}\text { oce que las matemáticas sirven para desenvolverse en la vida y que } \\
\text { el estudio de otras ciencias }\end{array}$ \\
\hline$\%$ & & e, a & S, que las matemáticas sirven para desenvolverse en la vida \\
\hline
\end{tabular}

Figura 5. Escalera de aprendizaje de la pregunta 1: ¿Por qué se enseña Matemáticas?

\begin{tabular}{|c|c|c|c|c|}
\hline & & & $\begin{array}{l}\text { I: } 0 \% \\
F: 20 \%\end{array}$ & $\begin{array}{l}\text { N4: Además de las anteriores, reconocen la finalidad } \\
\text { politica (como consecuencias éticas y sociales) }\end{array}$ \\
\hline & & $\begin{array}{l}\text { 1: } 3 \% \\
\text { F: } 70 \%\end{array}$ & $\begin{array}{l}\text { N3: Ade } \\
\text { cultural }\end{array}$ & $\begin{array}{l}\text { de la finalidad socialy formativa, reconoce la finalidad } \\
\text { herencia de cada sociedad }\end{array}$ \\
\hline $90 \%$ & F: $10 \%$ & $\begin{array}{l}\text { N2: Adem } \\
\text { (desarroll }\end{array}$ & $\begin{array}{l}\text { ás de la fi } \\
\text { y adquis }\end{array}$ & $\begin{array}{l}\text { idad social, reconoce que pueden tener finalidad formativa } \\
\text { ón de capacidades) }\end{array}$ \\
\hline $0 \%$ & cono & $\begin{array}{l}\text { la fina } \\
\text { matem }\end{array}$ & $\begin{array}{l}\text { d socia } \\
\infty 0)\end{array}$ & las matemáticas (finalidad de carácter utilitario del \\
\hline
\end{tabular}

Figura 6. Escalera de aprendizaje de la pregunta 2: ¿Se pueden apreciar diferentes fines (o finalidades) en la Educación Matemática?

\footnotetext{
I: $0 \% \quad$ N3: Aceptan que existen distintas visiones y que estas notienen por F: $20 \%$ qué ser opuestas

I. $0 \%$ N2: Son conscientes de que hay distintos tipos de considerar el curriculum F: $80 \%$ N2: Son consín etnomatemáticas) N1: No conocen que existan diferentes formas de considerar el curriculum
}

Figura 7. Escalera de aprendizaje de la pregunta 3: ¿Pueden existir distintas formas de considerar el currículo matemático? 


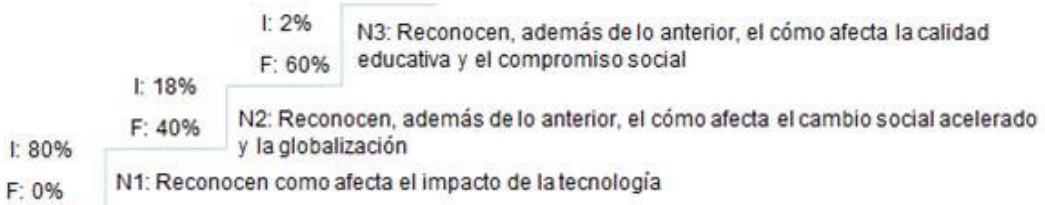

Figura 8. Escalera de aprendizaje de la pregunta 4: ¿Qué se puede decir sobre el 'curriculum del futuro' en relación con las Matemáticas?

Con respecto a las escaleras de aprendizaje en la pregunta 1 (figura 5) podemos resaltar que la mayoría de los estudiantes consiguió sin dificultad llegar al nivel máximo esperado. Sin embargo, en el resto de preguntas si se observan algunas dificultades de aprendizaje en la adquisición de los distintos niveles establecidos. En la pregunta 2 (figura 6) establecimos 4 niveles debido a la complejidad de ésta, quedando la mayoría de ellos en el N3 y solo unos pocos alcanzando el nivel máximo. En la pregunta 3 (figura 7) ocurre algo parecido a la pregunta 2, aunque de ahí solo establecimos 3 niveles. Y por último, en la pregunta 4 (figura 8) los estudiantes quedan repartidos entre los niveles 2 y 3 con lo que observamos un obstáculo de aprendizaje patente al no considerar cómo afecta la calidad educativa o el compromiso social a lo que es el curriculum del futuro en relación con las matemáticas.

\section{Evaluación del diseño puesto en práctica}

Como hemos comentado anteriormente, además de evaluar el aprendizaje de nuestros estudiantes, no podemos olvidar nuestra puesta en práctica. En este sentido, hay que comentar que para mí la valoración de la experiencia, una vez más, ha sido gratificante, al observar como todos han aprendido y sentir que he conectado con los estudiantes. Además, tengo que admitir que me ha servido bastante el recabar esas ideas previas de los estudiantes para poder ir armando las preguntas encadenadas para 
Llegar a la solución de cada pregunta estructurante. Con respecto a los tiempos comentar que para mi próximo ciclo de mejora intentaré ajustarme mejor los tiempos. Como queda reflejado en la aplicación del CIMA no se ajusta a lo que es el diseño que tenía establecido. La dificultad surgió en el primer módulo, que se ha excedido una semana más de lo que tenía establecido. Y en el resto, pues porque reducimos el contenido de las preguntas, así que como propósito para el próximo CIMA ajustar mejor los tiempos en las secuencias de actividades.

Para evaluar el aprendizaje de los estudiantes, como siempre, me han sido de gran utilidad las escaleras de aprendizaje, ya que nos muestran la evolución del aprendizaje de estos, que aunque no podamos decir que todos los alumnos han alcanzado en nivel más elevado que se pretendía, lo que sí podemos afirmar es que todos han logrado una evolución positiva.

Como comentaba al inicio de este apartado, también les pedía que evaluaran mi práctica. Este cuestionario fue el mismo que usé en el CIMA del curso pasado (Escudero-Domínguez, 2018), de tipo escala Likert, excepto la última pregunta que es de respuesta abierta, donde les preguntaba qué elementos creían que se podrían mejorar del proceso de enseñanza/ aprendizaje de esta asignatura. Los estudiantes han valorado positivamente esta forma de trabajar y comprender el contenido de los temas (80\%).

Jornadas de Formación e Innovación Docente del Profesorado | № 2 (2019) Esta obra se distribuye con la licencia Creative Commons 


\section{Referencias}

Bain, K. (2004). Lo que hacen los mejores profesores universitarios. Valencia: Universita de Valencia.

Carrillo, J., Climent, N., Gorgorió, N., Prat, M. y Rojas, F.. (2008). Análisis de secuencias de aprendizaje matemático desde la perspectiva de la gestión de la participación, Enseñanza de las Ciencias, 26(1)., 67-76.

Escudero-Domínguez, A. (2017). Ciclo de mejora en Recursos Didáctico Matemáticos en Educación Infantil. IV Jornadas de Formación e Innovación docente de la Universidad de Sevilla (pp. 483-493). Sevilla.

Escudero-Domínguez, A. (2018). Aprendiendo a enseñar Didáctica de las Matemáticas en el Grado de Educación Primaria. Monográfico Jornadas de Formación e Innovación docente del Profesorado 1, pp. 216-229. DOl.: http://dx.doi.org/10.12795/JDU.2018.i01.12 Pp.: 216-229

Finkel, D. (2008). Dar clase con la boca cerrada. Valencia: Universitat de Valencia.

Porlán, R. (Coord.). (2017). Enseñanza universitaria. Cómo mejorarla. Madrid: Ediciones

Morata.

Jornadas de Formación e Innovación Docente del Profesorado | № 2 (2019) Esta obra se distribuye con la licencia Creative Commons 